\title{
Erwiderung auf die Bemerkungen
}

F

ür Ihren anregenden Brief danken wir bestens. Sie sprechen darin vor allem an:

Das Standardinstrumentarium könnte auf Bullard-Laryngoskop, Larynxmasken aller Größen, Combitube und Miniconiotomie Set begrenzt werden.

Ohne Zweifel können sehr viele Fälle mit schwieriger Intubation durch Einsatz des Bullard-Laryngoskops (Abb. 1) schnell und elegant überwunden werden. In unserer begrenzten Erfahrung mit dem Bullard-Laryngoskop waren auch Patienten nach Kiefertumor-Operationen problemlos zu intubieren. International und nach eigener Erfahrung sind jedoch auch mit dem Bullard-Laryngoskop einige Situationen nicht zu meistern, z.B. absolute Kieferklemme, schwerer M. Bechterew oder überlanger Hals, so daß sich das Fiberbronchoskop als universellstes Mittel gegenüber dem Bullard-Laryngoskop durchgesetzt hat.

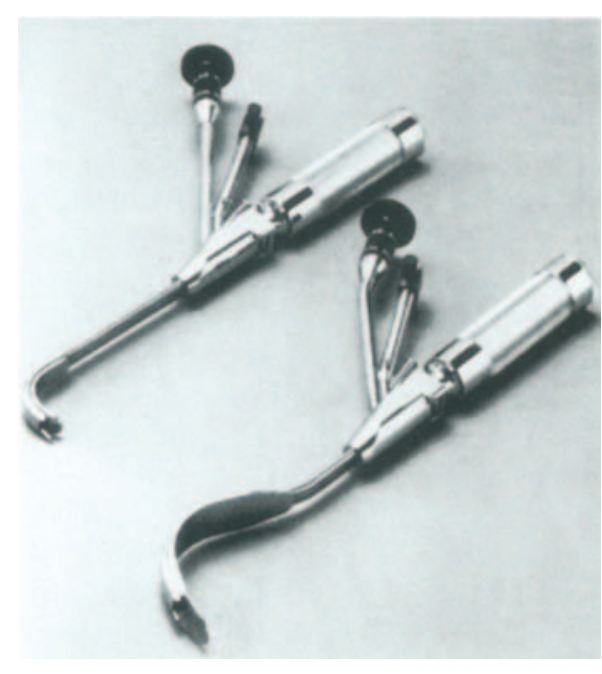

Die Wahl zwischen Bullard-Laryngoskop und fiberoptischem Bronchoskop ist vom Anschaffungspreis her schwierig (ca. 6000,- vs. DM 16000,plus jeweils Lichtquelle). Ich kenne kaum eine Abteilung, die über beide Mittel verfügt. Sie haben sicherlich recht darin, daß wir als Spezialisten für die Intubation die Anschaffung von zwei teuren Spezialinstrumenten nicht scheuen sollten.

In der Praxis stimme ich auch mit Ihnen überein, daß der überwiegende Anteil schwieriger Intubationen mit dem Bullard-Laryngoskop elegant zu lösen ist. Zusätzlich ist es erheblich robuster als das Bronchoskop, außerdem braucht man dann eine Vielfalt von speziellen Laryngoskopspateln nicht zusätzlich.

Inwieweit der Combitube Vorteile vor und neben der Larynxmaske bietet, können wir neben dem Hinweis, daß i.d.R. keine fiberoptische Intubations-

Abb. 1 Das ,Bullard"-Laryngoskop für Erwachsene (u) und Kinder (o). Der speziell gebogene Spatel wird mit einem normalen Laryngoskophandgriff eingeführt. An der Spatelspitze befindet sich die Spitze der Fiberoptik, durch die der Raum vor der Spatelspitze über das Okular betrachtet wird. Die Handhabung eines Tubus ist durch die extrem schlanke Höhe des „Bullard“Laryngoskops noch gut zu bewerkstelligen. Sehr viele Fälle von schwieriger Intubation sind mit dieser Spezialoptik problemlos zu lösen. möglichkeit besteht, nicht entscheiden, da wir ausschließlich die Larynxmaske verwenden.

Wir stimmen Ihnen ebenfalls voll zu, daß in verzweifelten Fällen ein Koniotomieset die wichtigste ,ultima ratio" Lösung in "cannot ventilate - cannot intubate"-Fällen darstellt mit den geschilderten Vor- und Nachteilen und unter der strikten Voraussetzung, daß der Anwender darin erhebliche Übung besitzt.

„Bochumer Standards“: Wegen allzu vielfältiger Ursachen für schwierige Intubation warnen wir vor zu enger Begrenzung auf wenige Hilfsmittel, sondern halten die ASA-Empfehlungen für sehr ausgewogen.

Unser „Standard“ ist: a) unbedingt pulsoxymetrische Überwachung bei schwierigen Intubationssituationen, b) alles Können einsetzen, um die Oxygenation zu erhalten (Drill in der Maskenbeatmung mit und ohne Hilfsmittel, Drill in der Handhabung der Larynxmaske, Einübung von Racheninsufflation von $\mathrm{O}_{2}$; Einübung von Jetventilation und Minikoniotomie an Leichen), c) bei gesicherter Oxygenation mit dem geeignetsten Mittel Herstellen eines sicheren Atemwegs, ohne Schaden zu verursachen, d) vor Start einer Narkose großzügige Entscheidung zur wachen Intubation, vor allem bei Halsschwellungen.

\section{Dr.H.Langenstein}

Klinik für Anaesthesie und Operative Intensivtherapie, Ruhr-Universität Bochum, Knappschaftskrankenhaus Bochum-Langendreer, In der Schornau 23, D-44892 Bochum 